\title{
Cilostazol suppresses angiotensin II-induced apoptosis in endothelial cells
}

\author{
MIAO-QIAN SHI ${ }^{1,2^{*}}$, FEI-FEI SU ${ }^{2 *}, \mathrm{XUAN} \mathrm{XU}^{1 *}, \mathrm{XIONG}^{*} \mathrm{TAO} \mathrm{LIU}^{2}, \mathrm{HONG}^{2} \mathrm{TAO} \mathrm{WANG}^{2}$, \\ WEI ZHANG ${ }^{2}$, XUE LI $^{2}$, CHENG LIAN ${ }^{2}$, QIANG-SUN ZHENG ${ }^{2}$ and ZHI-CHUN FENG ${ }^{1}$ \\ ${ }^{1}$ Department of Pediatrics, Affiliated Bayi Children's Hospital, General Military Hospital of Beijing PLA, \\ Beijing Key Laboratory of Pediatric Organ Failure, Beijing 100700; ${ }^{2}$ Department of Cardiology, \\ Tangdu Hospital, The Fourth Military Medical University, Xi'an, Shaanxi 710032, P.R. China
}

Received April 23, 2015; Accepted January 20, 2016

DOI: $10.3892 / \mathrm{mmr} .2016 .4881$

\begin{abstract}
Patients with essential hypertension undergo endothelial dysfunction, particularly in the conduit arteries. Cilostazol, a type III phosphodiesterase inhibitor, serves a role in the inhibition of platelet aggregation and it is widely used in the treatment of peripheral vascular diseases. Previous studies have suggested that cilostazol suppresses endothelial dysfunction; however, it remains unknown whether cilostazol protects the endothelial function in essential hypertension. The aim of the present study was to investigate whether, and how, cilostazol suppresses angiotensin II (angII)-induced endothelial dysfunction. Human umbilical vein endothelial cells (HUVECs) and Sprague Dawley rats were exposed to angII and treated with cilostazol. Endothelial cell apoptosis and function, nitric oxide and superoxide production, phosphorylation ( $\mathrm{p}$ ) of Akt, and caspase-3 protein expression levels were investigated. AngII exposure resulted in the apoptosis of endothelial cells in vitro and in vivo. In vitro, cilostazol significantly suppressed the angII-induced apoptosis of HUVECs; however, this effect was reduced in the presence of LY294002, a phosphoinositide 3 kinase (PI3K) inhibitor. Furthermore, cilostazol suppressed the angII-induced p-Akt downregulation and cleaved caspase-3 upregulation. These effects were also alleviated by LY294002. In vivo, cilostazol suppressed the angII-induced endothelial cell apoptosis and dysfunction. Cilostazol was also demonstrated to partially reduced the angII-induced increase in superoxide production. The results
\end{abstract}

Correspondence to: Professor Zhi-Chun Feng, Department of Pediatrics, Affiliated Bayi Children's Hospital, General Military Hospital of Beijing PLA, Beijing Key Laboratory of Pediatric Organ Failure, 5 Nanmencang Road, Dongcheng, Beijing 100700, P.R. China

E-mail: sufeifei830201@gmail.com

*Contributed equally

Key words: cilostazol, angiotensin II, phosphoinositide 3 kinase, Akt, endothelial cells of the present study suggested that cilostazol suppresses endothelial apoptosis and dysfunction by modulating the PI3K/Akt pathway.

\section{Introduction}

The endothelium lies in between the circulating blood and vascular smooth muscle cells, which are responsible for peripheral resistance (1). It may be easily damaged and endothelial dysfunction occurs in the pathogenesis of various cardiovascular complications, particularly in hypertension (2-6). Damage to the endothelium can result in a positive feedback mechanism as in arterial hypertension, as it negatively affects the vascular tone and homeostasis upon damage. Thus, it is an early independent predictor of cardiovascular events (7-10). Endothelial dysfunction contributes to an increase in large arterial stiffness in patients with isolated systolic hypertension, resulting in impaired vascular elasticity and compliance, and subsequent arterial hypertension $(11,12)$. Endothelial dysfunction increases the risk of developing atherosclerotic lesions and related cardiovascular events, even if the blood pressure of patients with essential hypertension is controlled (13). Restoration of conduit artery endothelial function is therefore a primary target in limiting cardiovascular morbidity and mortality in patients with essential hypertension (7).

Cilostazol, a type III phosphodiesterase inhibitor, inhibits platelet aggregation and it is widely used in the treatment of peripheral vascular diseases (14-16). Previous studies have demonstrated that cilostazol serves a role in the inhibition of endothelial cell apoptosis $(14,17)$. For example, it can suppress superoxide production and expression of adhesion molecules in human endothelial cells (18). In addition, cilostazol may prevent endothelial cell apoptosis by stimulating the extracellular signal-regulated kinase (ERK) 1/2 and p38 MAPK signaling, particularly in patients with hyperlipidemia and in pathological tissue conditions, including ischemia, shock and sepsis $(19,20)$. Previous studies have suggested that the phosphoinositide 3 kinase (PI3K)/Akt pathway serves an important role in preventing cell apoptosis induced by numerous stimuli $(21,22)$. In endothelial cells, PI3K/Akt activation promotes cell survival $(2,23)$. Previous studies have demonstrated that cilostazol produces a vasculo-angiogenic 
effect by upregulating a broad signaling network that includes the PI3K/Akt/endothelial nitric oxide synthase (eNOS) pathway (17). However, whether and how cilostazol protects the endothelial function in patients with essential hypertension remains unknown.

The aim of the present study was to determine whether cilostazol suppresses endothelial cell apoptosis and dysfunction in hypertension, and whether the PI3K/Akt pathway was involved. Evidence from previous studies suggest that angiotensin II (angII), a peptide of the rennin angiotensin system, exerts an vasoconstrictive effect, induces intracellular reactive oxygen species (ROS) production and causes vascular dysfunction and arterial hypertension (24-30). Therefore, Sprague Dawley (SD) rats were infused with angII to generate hypertension. The effect of cilostazol on endothelial function and apoptosis, as well as nitric oxide (NO) and superoxide production were investigated. Additionally, the PI3K/Akt signaling pathway was also investigated using human umbilical vein endothelial cells (HUVECs).

\section{Materials and methods}

Chemicals. Cilostazol, acetylcholine (Ach), sodium nitroprusside (SNP), angII and U46619 were gifts from Dr. Zhiqiang Yan (Department of Neurosurgery, Urumqi General Hospital of Lanzhou Military Command, Urumqi, China). LY294002 and 4',6-diamidino-2-phenylindole (DAPI) were gifts from Dr. Wei Zhang (Department of Cardiology, Tangdu Hospital, Fourth Military Medical University, Xi'an, China). The HUVEC cell line was a gift from Mr. Xiaofei Zhu (Department of Neurosurgery, Tangdu Hospital, Fourth Military Medical University).

Animal model and experimental design. Male SD rats (weight, 200-220 g; age, 10-12 weeks) were purchased from the Animal Center of The Fourth Military Medical University (FMMU). They were maintained in a temperature-controlled room $\left(24^{\circ} \mathrm{C}\right)$, on a $12 \mathrm{~h}$ light/dark cycle and given free access to food water. The experimental protocol was approved by the Institutional Care and Use Committee of the FMMU, which conforms to the Guidelines for the Care and Use of Laboratory Animals of the US National Institutes of Health (NIH publication no. 85-23, revised 1996) (31). Rats were divided into four groups ( $\mathrm{n}=10)$ : (i) Saline-treated group, rats treated with saline by intragastric administration (IA); (ii) Saline + Cilo-treated group, rats treated with saline and cilostazol (30 mg/kg/day) by IA; (iii) angII-treated group, rats continuously infused with angII (1,000 ng/kg/min) by subcutaneously implanted Alzet osmotic pumps (2004 model; Cupertino,CA,USA) as previously described (32); and (iv) angII (1,000 ng/kg/min) + Cilo-treated group, rats treated with cilostazol $(30 \mathrm{mg} / \mathrm{kg} /$ day $)$ in addition to $\operatorname{angII}(1,000 \mathrm{ng} / \mathrm{kg} / \mathrm{min})$ infusion.

After 4 weeks of angII infusion and cilostazol administration, rats were anesthetized with an intraperitoneal injection of sodium pentobarbital (45 mg/kg; Shanghai Rongbai Biological Technology Co., Ltd., Shanghai, China). Hemodynamic measurements were obtained as previously described (33). In brief, the right carotid artery of anesthetized rats was cannulated with a catheter connected to a microtip pressure transducer (Chengdu Instrument Factory, Chengdu, China) and the transducer was connected to a recording system (Rm6280C, Biological Instruments). The systolic and diastolic blood pressure (sBP and dBP, respectively) were then measured. Finally, the rats were sacrificed by cervical vertebral dislocation. The abdominal aortae were removed and divided into two sections by transverse section. The first was incubated in $10 \%$ buffered formalin (Sangon Biotech Co., Ltd., Shanghai, China) for $24 \mathrm{~h}$ and then paraffin-embedded (Sangon Biotech Co., Ltd.) for further assessment of apoptosis. The second was used for the detection of endothelium-dependent and -independent vasorelaxation, and assessment of superoxide anion and NO production.

Detection of endothelium-dependent and independent vasorelaxation. Abdominal aortae were carefully dissected and mounted as ring preparations of $\sim 3 \mathrm{~mm}$ on hooks in individual organ baths (Radnoti Glass Technology, Monrovia, CA, USA). Arterial integrity was assessed first by stimulation of vessels with $120 \mathrm{mM} \mathrm{KCl}$, and then washed three times in physiological salt solution $(130 \mathrm{mM} \mathrm{NaCl}, 14.9 \mathrm{mM} \mathrm{NaHCO} 3,4.7 \mathrm{mM}$ $\mathrm{KCl}, 1.18 \mathrm{mM} \mathrm{KH} \mathrm{PO}_{4}, 1.18 \mathrm{mM} \mathrm{MgSO} \mathrm{m}_{4} 7 \mathrm{H}_{2} \mathrm{O}, 1.56 \mathrm{mM}$ $\mathrm{CaCl}_{2} \mathrm{H}_{2} \mathrm{O}, 0.026 \mathrm{mM}$ EDTA and $5.5 \mathrm{mM}$ glucose). The temperature was maintained at $37^{\circ} \mathrm{C}$ and $95 \% \mathrm{O}_{2}$ and $5 \% \mathrm{CO}_{2}$ was pumped into the physiological salt solution. Following washing and stabilization, by contracting the segments with phenylephrine $(10 \mu \mathrm{mol} / \mathrm{l})$, followed by relaxation with Ach (10 $\mu \mathrm{mol} / \mathrm{l})$. The contraction response was detected using an organ chamber containing an isometric Mulvany-Halpern myograph (model 610; DMT-USA, Inc., Marietta, GA, USA) and recorded using a PowerLab 8/SP data acquisition system (ADInstruments Ltd., Colorado Springs, CO, USA), as previously described (34-36). Contractile responses of abdominal aortic rings were evoked with $30 \mathrm{nmol} / 1 \mathrm{U} 46619$. At the plateau of contraction, Ach $\left(1 \times 10^{-8}-1 \times 10^{-4} \mathrm{~mol} / \mathrm{l}\right)$ or SNP $\left(1 \times 10^{-10}-1 \times 10^{-6} \mathrm{~mol} / \mathrm{l}\right)$ were progressively added to the organ bath to induce endothelium-dependent or -independent relaxation.

Detection of superoxide anion production. Superoxide anions from the aortae were measured using flow injection chemiluminescence, as previously described (37). The superoxide anion concentration is reported as chemiluminescence intensity (CI) per mg of tissue weight.

Detection of total NO production. The total NO production in aortae was determined by measuring nitrite concentration, as previously described (37). The concentration of nitrite was calculated from a nitrite standard curve.

Immunohistochemistry. Tissue samples were prepared from abdominal aortae. After being paraffin-embedded, the abdominal aorta was exposed by a transverse section and cut into $4 \mu \mathrm{m}$ thick sections. The cell nuclei of the sections were stained with DAPI as previously described (26). The immunofluorescence data were analyzed using an Eclipse Ni-E microscope (Nikon, Tokyo, Japan) and NIS-elements imaging software (Nikon).

Cell culture and treatment. HUVECs were provided by Dr. Xiaofei Zhu from the Department of Neurosurgery, 
Table I. Comparison of blood pressure among the four groups of treated rats.

\begin{tabular}{lcr}
\hline Group & sBP $(\mathrm{mmHg})$ & $\mathrm{dBP}(\mathrm{mmHg})$ \\
\hline Saline & $125.8 \pm 5.3$ & $78.2 \pm 5.9$ \\
Saline+Cilo & $125.7 \pm 6.0$ & $81.3 \pm 2.3$ \\
AngII & $192.5 \pm 4.6^{\mathrm{a}}$ & $111.8 \pm 6.3^{\mathrm{a}}$ \\
AngII+Cilo & $193.5 \pm 3.4^{\mathrm{a}}$ & $114.3 \pm 4.6^{\mathrm{a}}$
\end{tabular}

AngII infusion increases blood pressure in rats. The sBP and dBP in the four groups of rats treated with angII, Cilo and/or saline (as a control) for 4 weeks is demonstrated. Data are expressed as the mean \pm standard error of the mean $(n=6)$. ${ }^{a} \mathrm{P}<0.05$ vs. the saline group. sBP, systolic blood pressure; dBP, diastolic blood pressure; Cilo, cilostazol; AngII, angiotensin II.

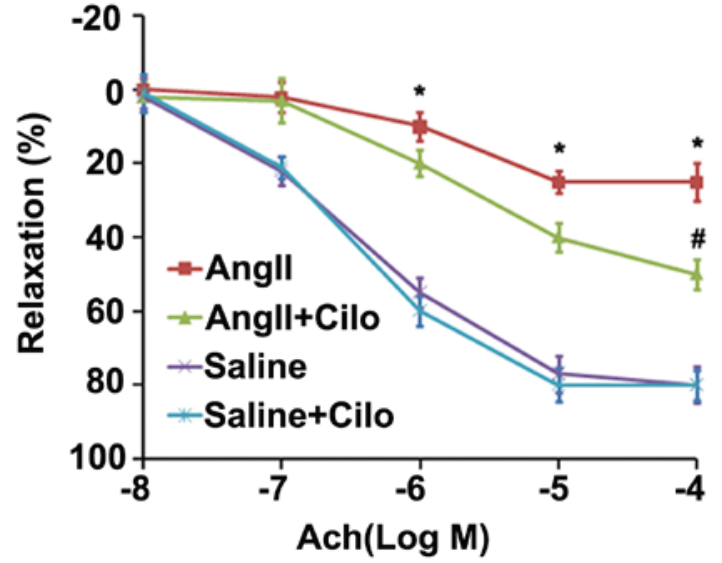

Figure 1. Effect of cilostazol on Ach-induced endothelium-dependent relaxation. The logarithmic plot demonstrates that angII impaired endothelial function by acting on endothelial relaxation. Cilostazol partially rescued the endothelial dysfunction induced by angII $(n=6)$. ${ }^{*} \mathrm{P}<0.05$ vs. the saline group and ${ }^{\#} \mathrm{P}<0.05$ vs. the angII group. AngII, angiotensin II; Cilo, cilostazol; Ach, acetylcholine.

Tangdu Hospital, Fourth Military Medical University (Xi'an, China). HUVEC monolayers were grown as previously described $(2,20)$. In brief, cells were plated into dishes with Gibco Dulbecco's Modified Eagle's medium (Thermo Fisher Scientific, Inc., Waltham, MA, USA) containing 1\% penicillin-streptomycin (Beyotime Institute of Biotechnology, Shanghai, China) and 10\% fetal bovine serum (Beyotime Institute of Biotechnology), under $5 \% \mathrm{CO}_{2}$ at $37^{\circ} \mathrm{C}$, at a density of $1 \times 10^{5}$ cells $/ \mathrm{ml}$. Cells were supplemented with $5 \mathrm{U} / \mathrm{ml}$ heparin (Sangon Biotech Co., Ltd.) and $100 \mathrm{ng} / \mathrm{ml}$ endothelial cell growth substance (Collaborative Research Inc., Bedford, MA, USA). When the cells reached confluence (90\%), subcultures were prepared. Cells in actively growing conditions between the third and fifth passages were used for further experiments.

HUVECs were divided into four parallel groups: Untreated cells (control group); cells treated with $10 \mu \mathrm{mol} / 1$ angII (angII-treated group); cells pretreated with $10 \mu \mathrm{mol} / 1$ cilostazol prior to incubation with angII (angII + Cilo-treated group); and cells pretreated with a combination of cilostazol and LY294002 (a PI3K inhibitor) prior to incubation with angII (angII + Cilo + LY-treated group). The concentrations of cilostazol and angII were selected on the basis of previous studies $(20,24)$.
Immunocytochemistry. Cultured HUVECs were fixed with $10 \%$ buffered formalin and permeabilized with $0.5 \%$ Triton X-100 (Sangon Biotech Co., Ltd.). The cell nuclei were stained with DAPI as previously described (33).

Western blot analysis of HUVECs. Cultured HUVECs in lysis buffer containing $50 \mathrm{mmol} / 1$ Tris-hydrochloride, $150 \mathrm{mmol} / \mathrm{l}$ sodium chloride, $1 \%$ Nonidet P-40, $0.25 \%$ superoxide dismutase, $1 \mathrm{mmol} / 1$ EDTA, $1 \mathrm{mmol} / 1 \mathrm{NaF}, 1 \mathrm{mmol} / 1$ $\mathrm{Na}_{3} \mathrm{VO}_{3}, 1 \mathrm{mM}$ phenylmethylsulfonyl fluoride and a proteinase inhibitor cocktail tablet (Roche Diagnostics, Basel, Switzerland). Protein samples were assessed by sodium dodecyl sulfate-polyacrylamide gel electrophoresis, as described previously (33). In brief, total protein concentration of each sample was determined prior to polyacrylamide gel electrophoresis, followed by the transfer of proteins to polyvinylidene fluoride (PVDF) membranes (Sangon Biotech Co., Ltd.). PVDF membranes were incubated with blocking buffer (LI-COR Biosciences, Lincoln, NE, USA) for $1 \mathrm{~h}$ at room temperature and subsequently immunoblotted with either of the following primary antibodies: Polyclonal rabbit total Akt (1:1,000; cat. no. SAB4500797; Sigma-Aldrich, St. Louis, MO, USA); polyclonal rabbit phosphorylated-Akt (p-Akt) $(1: 1,000$; cat. no. SAB4504017; Sigma-Aldrich); cleaved and total monoclonal rabbit caspase-3 (1:1,000; cat. no. 9665; Cell Signaling Technology, Inc., Danvers, MA, USA); and monoclonal mouse $\beta$-actin (1:10,000; cat. no. A5441; Sigma-Aldrich). $\beta$-actin protein expression served as a loading control. Following being immunoblotted with primary antibodies overnight at $4^{\circ} \mathrm{C}$, the PVDF membranes were washed three times $(10 \mathrm{~min}$ each) in Tris-buffered saline containing Tween-20 and then incubated with IRDye 680RD goat anti-rabbit IgG (1:5,000; cat. no. 925-68071; LI-COR Biosciences) or IRDye $800 \mathrm{CW}$ goat anti-mouse IgM (1:5,000; cat. no. 926-32280; LI-COR Biosciences) for $60 \mathrm{~min}$ at room temperature, separately. The PVDF membranes were then washed three times $(10 \mathrm{~min}$ each) in phosphate-buffered saline with Tween 20. Bands were evaluated by densitometry using an Odyssey infrared imaging system (LI-COR Biosciences, Lincoln, NE, USA).

Terminal deoxynucleotidyl transferase dUTP nick end labeling (TUNEL) assay of HUVECs and abdominal aorta of endothelial cells. The TUNEL assay kit (Beyotime Institute of Biotechnology) was used to detect apoptotic cells according to the manufacturer's instructions. TUNEL-positive cells were 


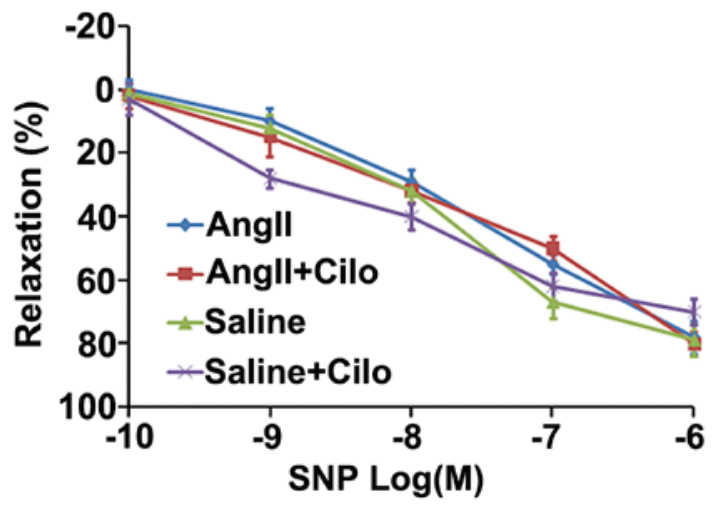

Figure 2. Effect of cilostazol on SNP-induced endothelium-independent relaxation. AngII, angiotensin II; Cilo, cilostazol; SNP, sodium nitroprusside.

detected by microscopy (Eclipse Ni-E; Nikon). The apoptotic index is expressed as the number of positively stained cells per total number of endothelial cells.

Statistical analysis. Data are presented as the mean \pm standard error of the mean. SPSS 13.0 software (SPSS, Inc, Chicago, IL, USA) was used to analyze the data. One-way analysis of variance was used to determine statistically significant differences among the four groups. $\mathrm{P}<0.05$ was considered to indicate a statistically significant difference.

\section{Results}

AngII-infusion increases the $S B P$ and $d B P$ levels in treated rats. Previous studies have demonstrated that angII infusion at doses between 175 and $1,000 \mathrm{ng} / \mathrm{kg} / \mathrm{min}$ may result in hypertension in rats $(32,38,39)$. In order to demonstrate the hypertensive effect of angII, a dose of $1,000 \mathrm{ng} / \mathrm{kg} / \mathrm{min}$ was infused into rats for the period of 4 weeks. Following the infusion, the sBP and dBP levels were measured and demonstrated to be significantly increased compared with the saline-treated group ( $\mathrm{P}<0.05$; Table I). Cilostazol was not identified to exhibit an effect on angII-infusion-dependent hypertension (Table I).

Cilostazol treatment inhibits angII-induced dysfunction and apoptosis of endothelial cells. Evidence from previous studies has suggested that angII induces aberrant oxidative stress in the vascular wall and, therefore, intracellular ROS production, resulting in excessive apoptosis and dysfunction of the epithelium and endothelium (40-42). Whether cilostazol suppresses angII induced endothelial dysfunction remains unknown. Thus, the percentage of Ach-induced vascular relaxation was investigated (Fig. 1). Compared with the saline-treated rats, abdominal aorta rings from the angII-infused rats demonstrated significantly impaired Ach-induced endothelium-dependent relaxation $\left({ }^{*} \mathrm{P}<0.05\right.$; Fig. 1$)$. Cilostazol significantly reduced the impairment in vasorelaxation in the angII +Cilo-treated group compared with the angII-treated group $\left({ }^{*} \mathrm{P}<0.05 ;\right.$ Fig. 1$)$. No significant difference among the four groups of rats was identified upon investigation of SNP-induced endothelium-independent relaxation (Fig. 2).

Furthermore, the apoptosis of endothelial cells was investigated. Compared with the saline-treated group, endothelial
A
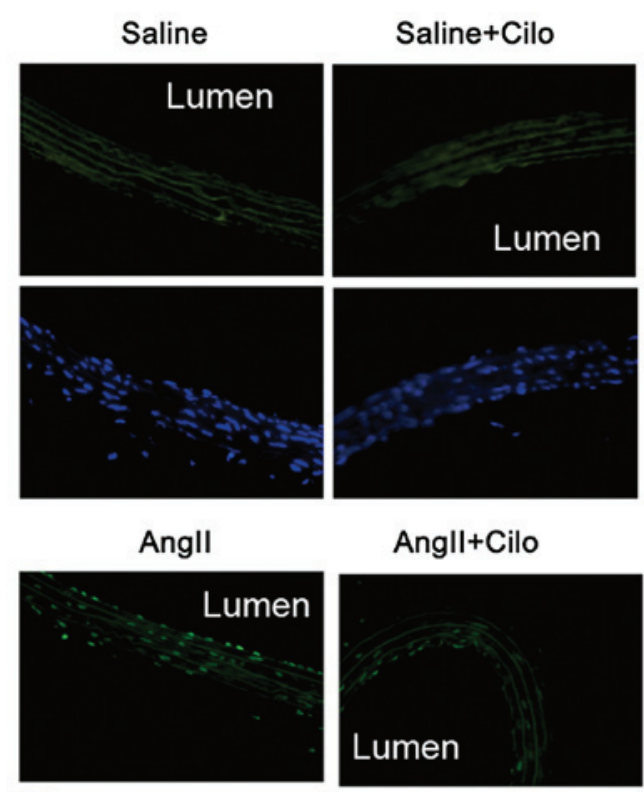

Angll+Cilo
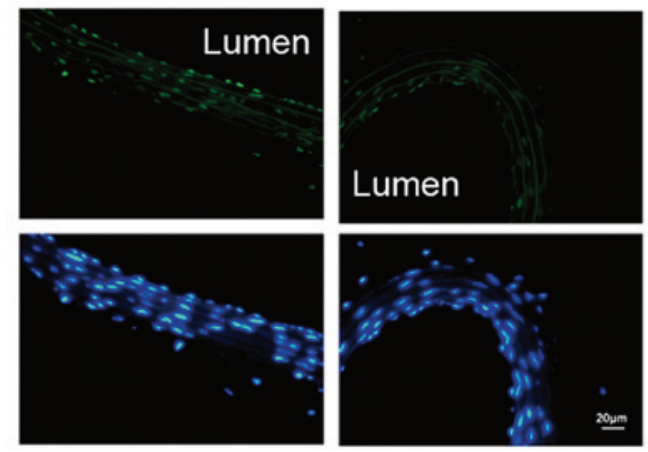

B

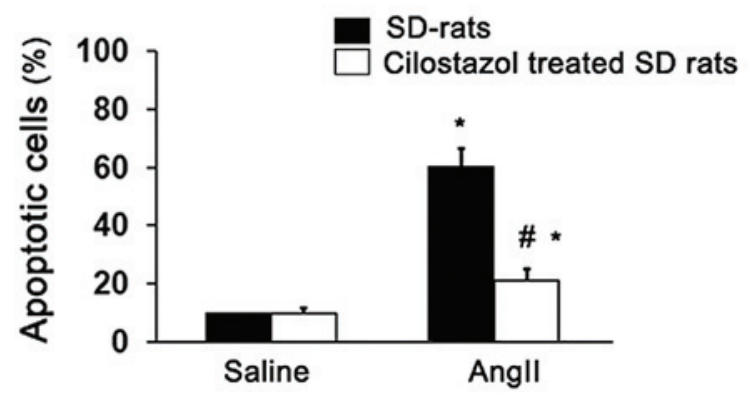

Figure 3. Effect of cilostazol on angII-induced apoptosis in vivo. The TUNEL assay was used to detect the apoptotic endothelial cells. (A) Representative images of TUNEL stained endothelial cells. Green fluorescence represents TUNEL-positive cells and blue (DAPI) the cell nuclei. (B) Quantitative analysis of the imaging data. The number of apoptotic cells is presented as the percentage of total endothelial cells (mean \pm standard error of mean; $\mathrm{n}=6) .{ }^{*} \mathrm{P}<0.05$ vs. the saline-treated group and ${ }^{\#} \mathrm{P}<0.05$ vs. the angII-treated group. Cilo, cilostazol; AngII, angiotensin II; SD, Sprague Dawley; TUNEL, terminal deoxynucleotidyl transferase dUTP nick end labeling.

apoptosis was significantly increased in the angII-infused rats (" $\mathrm{P}<0.05$; Fig. 3). Endothelial apoptosis was significantly decreased in angII-infused rats treated with cilostazol compared with the angII-treated group $\left({ }^{\#} \mathrm{P}<0.05\right.$; Fig. 3$)$. The cilostazol + saline-treatment had no effect on endothelial apoptosis (Fig. 3) or the Ach-induced vasorelaxation (Fig. 1). These results suggest that cilostazol alleviates the endothelial dysfunction in angII-induced hypertension rats, possibly through an anti-apoptotic effect.

Effect of cilostazol on the angII-induced increase in superoxide anion production. AngII acts via the nicotinamide adenine dinucleotide phosphate (NADPH) oxidase-derived 


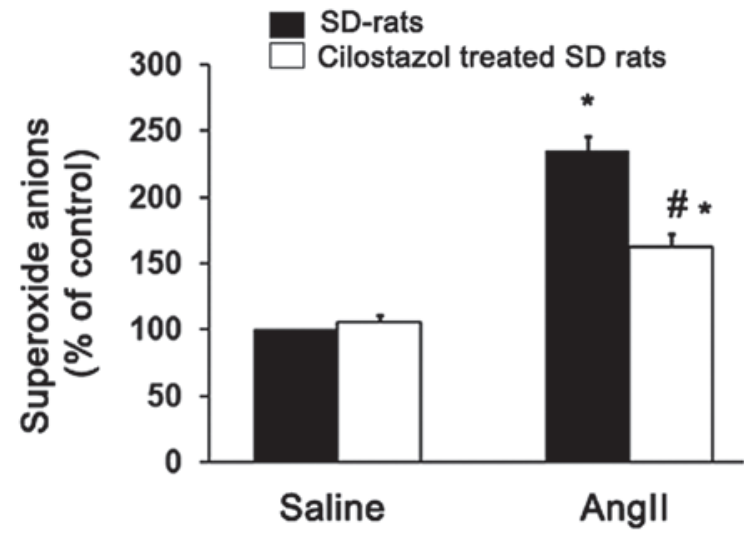
induced an increase in the aortic superoxide anion production in vivo and cilostazol partially compensated for this effect. ${ }^{*} \mathrm{P}<0.05 \mathrm{vs}$. the saline-treated group and ${ }^{\#} \mathrm{P}<0.05$ vs. the angII-treated group. SD, Sprague Dawley; AngII, angiotensin II.
Figure 4. Effect of cilostazol on the production of superoxide anions. AngII
A
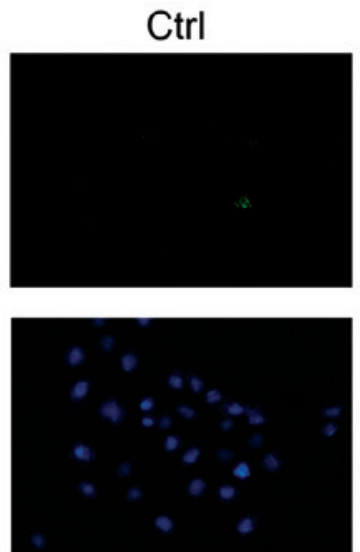

Angll+Cilo
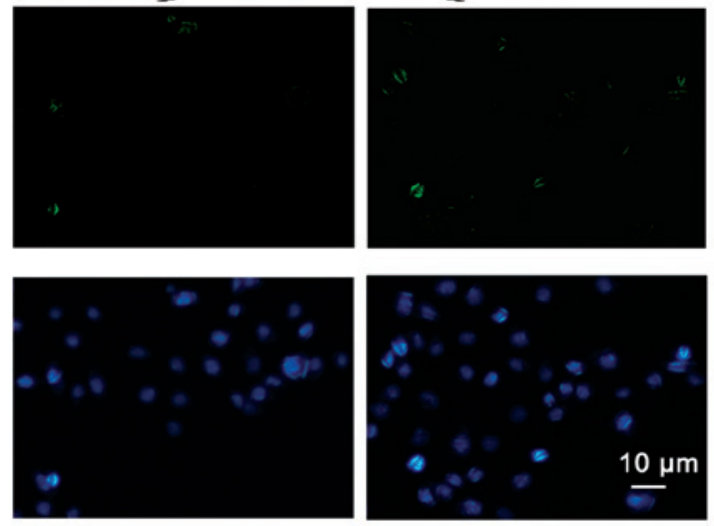

B
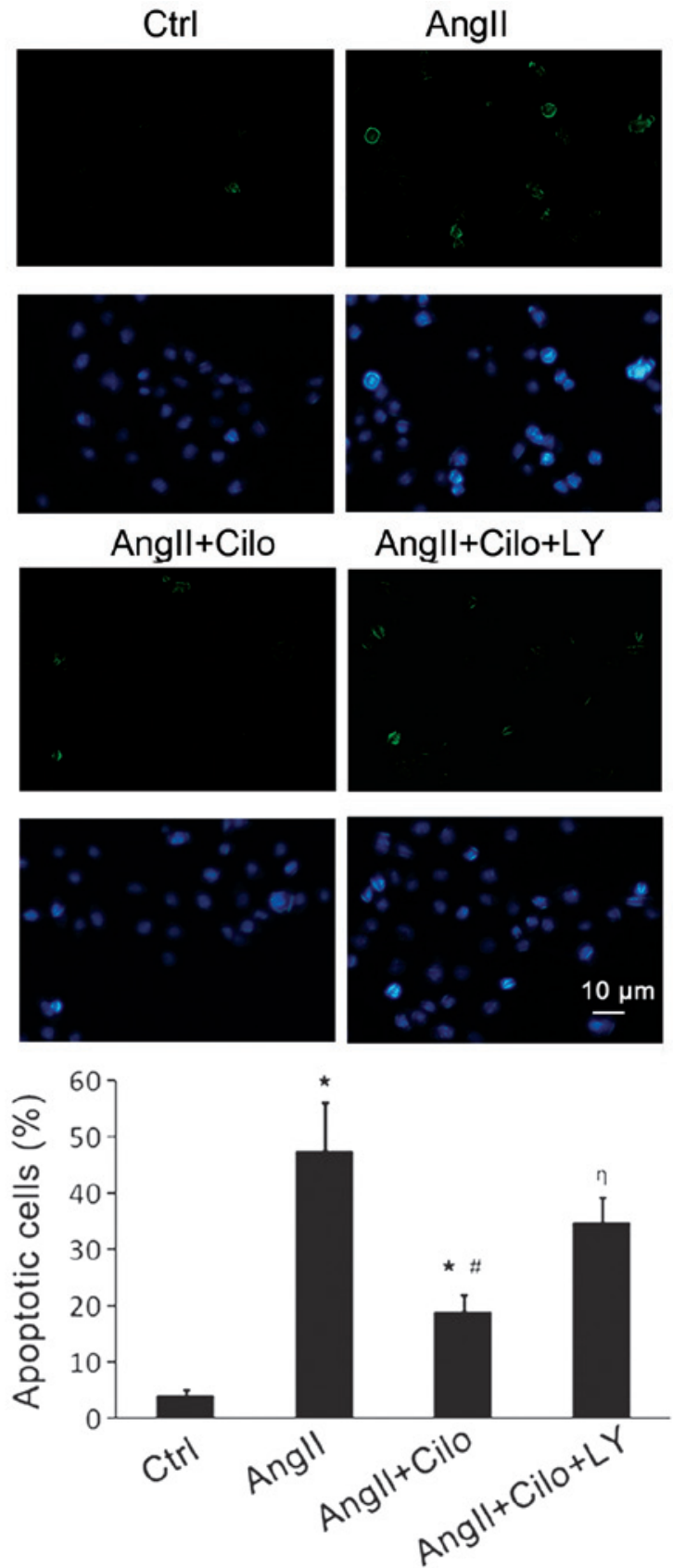

ROS to trigger endothelial cell apoptosis and impair endothelium-dependent relaxation (34). Cilostazol may therefore alleviate endothelial cell apoptosis and attenuate impairment in vasorelaxation by inhibiting superoxide production. Thus, the superoxide levels produced in the aortic tissue from the four groups of treated rats were determined and compared. AngII significantly increased the superoxide production compared with the saline-treated group ( $\mathrm{P}<0.05$; Fig. 4). However, cilostazol significantly suppressed the superoxide anion production compared with the angII-only treated group $\left({ }^{\#} \mathrm{P}<0.05\right.$; Fig. 4).

Effect of cilostazol on NO production. To detect whether cilostazol has an effect on NO production and thus improves vasorelaxation in response to Ach, total NO production in aortae was determined from the concentration of nitrite, a stable metabolite of NO in vitro (38). AngII significantly increased the total NO production compared with the saline-treated, control group ( ${ }^{*} \mathrm{P}<0.05$; Fig. 5). Cilostazol had no effect on the angII-induced NO production. (1)

Figure 5. Effect of cilostazol on NO production (demonstrated as nitrite compared with the saline-treated group. Cilostazol had no effect on the angII-induced NO increase. ${ }^{*} \mathrm{P}<0.05$ vs. the saline-treated group. SD, Sprague Dawley; AngII, angiotensin II; NO, nitric oxide.

.

Figure 6. Effect of cilostazol on the angII-induced apoptosis of HUVECs. The TUNEL assay was used to detect apoptotic HUVECs. (A) Representative images of TUNEL stained cells. Green fluorescence represents TUNEL-positive cells and blue (DAPI) indicates the cell nuclei. (B) Quantitative analysis of the cell images. The number of apoptotic cells is presented as the percentage of the total cells (mean \pm standard error of the mean; $n=6)$. ${ }^{*} \mathrm{P}<0.05$ vs. the $\mathrm{Ctrl}$ group; ${ }^{\text {}} \mathrm{P}<0.05$ vs. the angII-treated group; ${ }^{\eta} \mathrm{P}<0.05$ vs. the angII + Cilo-treated group. Ctrl, saline-treated group; AngII, angiotensin II-treated group; AngII + Cilo, cells pretreated with $10 \mu \mathrm{M}$ cilostazol prior to incubation with angiotensin II; AngII + Cilo + LY, cells pretreated with a combination of cilostazol and LY294002 prior to incubation with angiotensin II; TUNEL, terminal deoxynucleotidyl transferase dUTP nick end labeling.

Inhibition of angII-induced HUVEC apoptosis by cilostazol. AngII treatment $(10 \mu \mathrm{mol} / \mathrm{l})$ resulted in a significant increase in the number of TUNEL-positive (apoptotic) HUVECs compared with the control group ( $\mathrm{P}<0.05$; Fig. 6). Pretreatment with cilostazol $(10 \mu \mathrm{mol} / \mathrm{l})$ reduced the number of TUNEL-positive HUVECs produced on exposure to angII, compared with the 


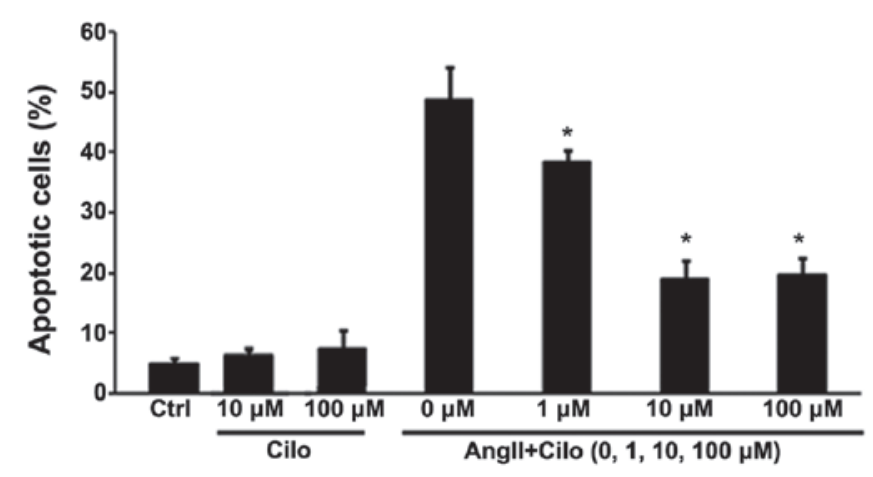

Figure 7. Effect of various concentrations of cilostazol on the apoptosis of HUVECs. The number of apoptotic cells is presented as the percentage of the total cells (mean \pm standard error of mean; $n=6$ ). ${ }^{*} \mathrm{P}<0.05$ vs. the Cilo-treated group. Ctrl, saline-treated group; Cilo, cilostazol-treated group; AngII + Cilo, cells pretreated with $0,1,10$ or $100 \mu \mathrm{M}$ cilostazol prior to incubation with $10 \mu \mathrm{M}$ angiotensin II.

angII-treated group ( ${ }^{\#} \mathrm{P}<0.05$; Fig. 6). LY294002, a specific inhibitor of PI3K, was used to detect whether the PI3K/Akt pathway was involved in the effect of the cilostazol treatment. Compared with the angII + Cilo-treated group, HUVECs pretreated with a combination of cilostazol and LY294002 demonstrated increased numbers of TUNEL-positive cells ( ${ }^{\mathrm{P}}<0.05$; Fig. 6).

Cilostazol $(1,10$ and $100 \mu \mathrm{mol} / \mathrm{l})$ was administered to HUVECs to investigate the effect concentration. Cilostazol at $1 \mu \mathrm{mol} / 1$ mildly inhibited angII-induced HUVEC apoptosis, compared with concentrations of 10 or $100 \mu \mathrm{mol} / 1$ which significantly attenuated apoptosis, to similar levels, in the angII treated group ( ${ }^{*} \mathrm{P}<0.05 ;$ Fig. 7$)$.

Effect of cilostazol on Akt and cleaved caspase-3 protein expression levels in HUVECs. To elucidate the mechanism underlying the cilostazol-dependent reduction of angII-induced apoptosis, the effect of cilostazol on Akt phosphorylation was examined using western blot analysis (Fig. 8A). As demonstrated in Figure 8B, angII significantly reduced the phosphorylation of Akt compared with the control group $\left({ }^{*} \mathrm{P}<0.05\right)$ and cilostazol attenuated the reduction of Akt phosphorylation compared with the angII-treated group $\left({ }^{\#} \mathrm{P}<0.05\right)$. Furthermore, the effect of cilostazol on Akt phosphorylation was reduced by combinational treatment with LY294002 compared with the angII + Cilo-treated group $\left({ }^{n} \mathrm{P}<0.05\right.$; Fig. 8B).

AngII treatment upregulated the cleaved caspase-3 protein expression levels compared with the control group $\left({ }^{*} \mathrm{P}<0.05\right.$; Fig. 8C) and cilostazol treatment suppressed this effect compared with the angII-treated group $\left({ }^{\#} \mathrm{P}<0.05\right.$; Fig. 8C). LY294002 attenuated the effect of cilostazol on cleaved caspase- 3 protein expression compared with the angII + Cilo-treated group ("P $<0.05$; Fig. 8C).

\section{Discussion}

The results of the present study demonstrated that cilostazol suppressed the endothelial cell apoptosis induced by angII, in vivo and in vitro. In vivo, cilostazol suppressed the angII-induced endothelial dysfunction and increase

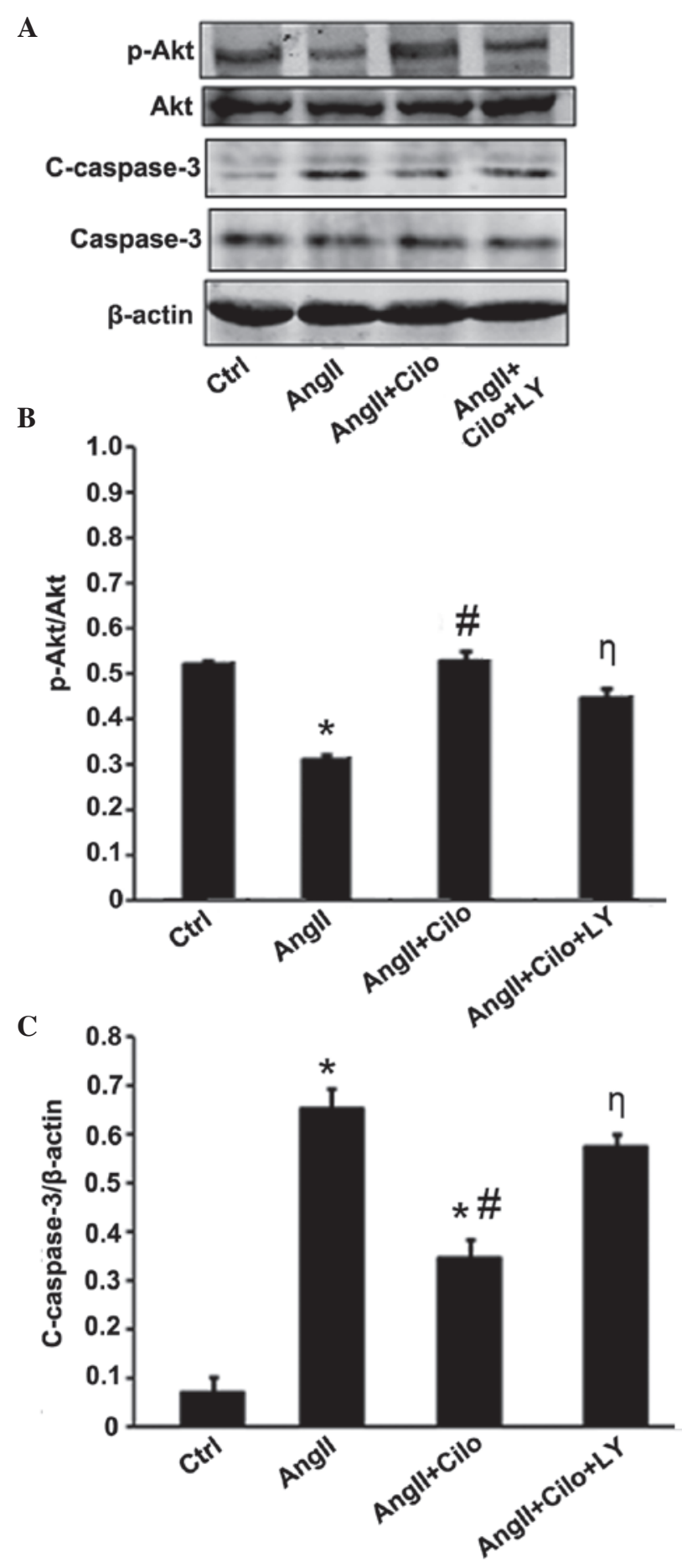

Figure 8. Western blot analysis of Akt, p-Akt and caspase-3 protein expression levels. $\beta$-actin was used as a loading control. (A) Representative western blots. Quantification of (B) Akt and p-Akt, and (C) cleaved caspase- 3 . Data are presented as the mean \pm standard error of the mean $(\mathrm{n}=3) .{ }^{*} \mathrm{P}<0.05$ vs. the Ctrl group, ${ }^{*} \mathrm{P}<0.05$ vs. the AngII-treated group and ${ }^{\eta} \mathrm{P}<0.05$ vs. the AngII + Cilo-treated group. p-Akt, phosphorylated-Akt; C-caspase-3, cleaved caspase-3; ctrl, saline-treated group; AngII, angiotensin II-treated group; AngII + Cilo, cells pretreated with $10 \mu \mathrm{M}$ cilostazol prior to incubation with angiotensin II; AngII + Cilo + LY, cells pretreated with a combination of cilostazol and LY294002 prior to incubation with angiotensin II.

in superoxide production, without affecting the total NO production. In vitro, cilostazol suppressed the angII-induced upregulation of cleaved caspase-3 protein levels and increase 


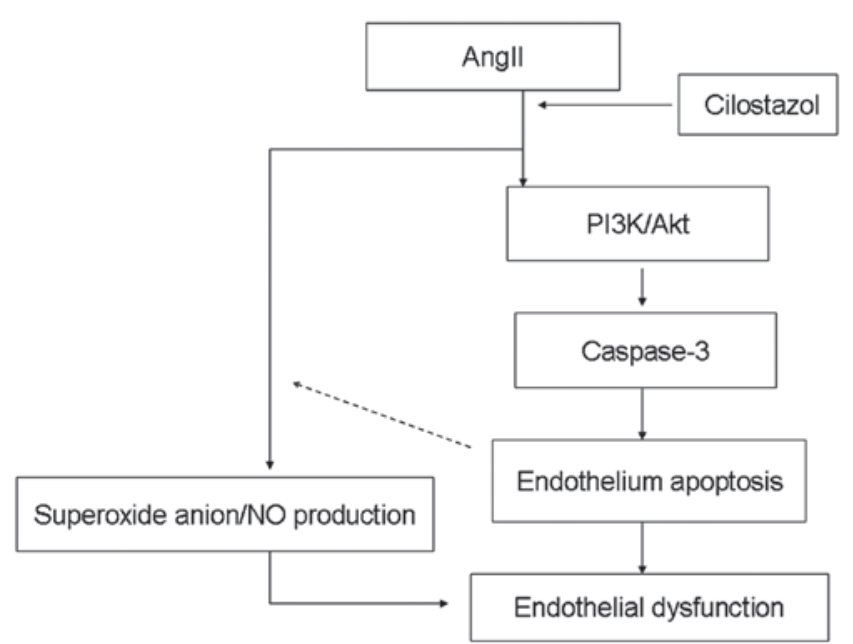

Figure 9. Proposed model of the effect of cilostazol on endothelial cell apoptosis and dysfunction. AngII treatment increased endothelial cell apoptosis and dysfunction. Cilostazol treatment suppressed the effect of angII by stimulating the PI3K/Akt pathway, inhibiting caspase-3, and regulating the balance between superoxide and NO production. Solid arrows demonstrate the effect demonstrated in the present study and the dashed arrow a possible pathway not yet verified. AngII, angiotensin II; PI3K, phosphoinositide 3 kinase; $\mathrm{NO}$, nitric oxide.

in apoptosis of HUVECs, and attenuated the angII-induced reduction of Akt phosphorylation. The effect of cilostazol on apoptotic HUVECs was blunted by LY294002, a PI3K inhibitor.

A previous study demonstrated that patients with essential hypertension suffer from endothelial dysfunction, particularly in the conduit arteries, due to damage from abnormal blood pressure (43). In turn, a damaged endothelium has a negative effect on the vascular tone, homeostasis and arterial stiffness. The endothelial dysfunction elevates the risk of cardiovascular events in patients with essential hypertension, whether the blood pressure is controlled or not (13). Endothelial cell apoptosis can induce endothelium dysfunction, therefore, its prevention may improve endothelial function and decrease the risk of cardiovascular disease (41). AngII is important in the pathogenesis of hypertension (44) and increased levels of angII promote the formation of atherosclerotic lesions (45). Previous experimental studies have indicated that atherosclerotic lesion-prone vascular regions are characterized by a high endothelial cell turnover, which has been attributed to an increased rate of endothelial cell apoptosis $(30,46)$. Thus, angII-induced endothelial cell apoptosis may serve an essential role in endothelial dysfunction in patients with essential hypertension. In the present study, angII was validated as a useful tool to induce hypertension and endothelial apoptosis in rats. The results of the present study demonstrated that angII treatment led to hypertension, endothelial dysfunction, an increase in superoxide production and endothelial apoptosis, consistent with previous studies $(39,47-50)$.

Cilostazol is a type III phosphodiesterase inhibitor and serves a role in the inhibition of endothelial cell apoptosis $(14,17)$. Thus, patients suffering from hypertension may benefit from administration of this drug, although the efficacy and mechanism of action in patients with hypertension have not yet been determined. In the present study, the effect of cilostazol on angII-induced (hypertensive) endothelial apoptosis and endothelial function was investigated. Cilostazol treatment suppressed the angII-induced endothelial dysfunction and apoptosis in vivo without affecting the blood pressure.

Vascular relaxation critically depends on the balance between superoxide and NO production by the vascular endothelium (51). Therefore, the superoxide anion and NO production was detected in the aortae of treated rats. Cilostazol attenuated the angII-induced increase in superoxide anion production, however had no effect on NO production. It may be considered controversial that angII increased the NO production and suppressed endothelial function, compared with cilostazol treatment which improved the endothelial function without affecting the NO production. However, these results may be due to the actions of different NO synthases (NOS), as endothelial NOS (eNOS) and inducible NOS (iNOS) serve different roles in the pathophysiology of cardiovascular diseases (52-54). Relatively low concentrations of NO appear to favor cell proliferation and anti-apoptotic responses compared with higher levels of NO which favor pathways inducing cell cycle arrest, mitochondrial respiration and apoptosis (55). Under pathological conditions increased amounts of NO are produced, resulting in stimulation of iNOS expression, and possibly endothelial dysfunction $(56,57)$. Further research is required to assess this effect.

In order to further investigate the mechanisms involved in the protective effects of cilostazol against endothelial apoptosis, HUVECs were utilized as an experimental tool. In vitro, cilostazol significantly reduced the angII-induced HUVEC apoptosis. Additionally, cilostazol attenuated the angII-induced reduction in Akt phosphorylation, and this protective effect of cilostazol on HUVEC apoptosis was inhibited by LY294002. The PI3K/Akt pathway is considered to be an important pathway for cell survival $(58,59)$, particularly in endothelial cells (2). Caspase-3 serves as a central member of the apoptotic cascade and can be activated to cleave the inhibitor of endonuclease, which cuts the DNA and induces the final stage of apoptosis. The present study demonstrated that angII treatment led to an upregulation of cleaved caspase- 3 and further treatment with cilostazol downregulated the cleaved caspase-3 in angII-treated cells.

In conclusion, cilostazol protects HUVECs from apoptosis by stimulating the PI3K/Akt pathway and inhibiting the caspase pathway. As indicated in Figure 9, the results of the current study suggest that cilostazol demonstrated a protective role against endothelial apoptosis by affecting the PI3K/AKt pathway and the superoxide anion/NO balance in animals suffering from angII-induced hypertension. Cilostazol may therefore represent a novel therapeutic agent for patients with essential hypertension.

\section{Acknowledgements}

The present study was supported by the National Natural Science Foundation of China (grant no. 81300077) and the 2012 Graduate Students Creativity Foundation of Tangdu Hospital, at The Fourth Military Medical University, China (grant no. 00543). 


\section{References}

1. Spieker LE, Flammer AJ and Lüscher TF: The vascular endothelium in hypertension. Handbook Exp Pharmacol 176: 249-283, 2006.

2. Zhang W, Wang R, Han SF, Bu L, Wang SW, Ma H and Jia GL: Alpha-linolenic acid attenuates high glucose-induced apoptosis in cultured human umbilical vein endothelial cells via PI3K/Akt/eNOS pathway. Nutrition 23: 762-770, 2007.

3. Fraga-Silva RA, Costa-Fraga FP, Murça TM, Moraes PL, Martins Lima A, Lautner RQ, Castro CH, Soares CM, Borges CL, Nadu AP, et al: Angiotensin-converting enzyme 2 activation improves endothelial function. Hypertension 61: 1233-1238, 2013.

4. Sun GB, Qin M, Ye JX, Pan RL, Meng XB, Wang M, Luo Y, Li ZY, Wang HW and Sun XB: Inhibitory effects of myricitrin on oxidative stress-induced endothelial damage and early atherosclerosis in ApoE-/- mice. Toxicol Appl Pharmacol 271: 114-126, 2013.

5. Guan Q, Zhang Y, Yu C, Liu Y, Gao L and Zhao J: Hydrogen sulfide protects against high-glucose-induced apoptosis in endothelial cells. J Cardiovasc Pharmacol 59: 188-193, 2012.

6. Park HS, Cho K, Park YJ and Lee T: Chronic nicotine exposure attenuates proangiogenic activity on human umbilical vein endothelial cells. J Cardiovasc Pharmacol 57: 287-293, 2011

7. Bellien J, Iacob M, Remy-Jouet I, Lucas D, Monteil C, Gutierrez L, Vendeville C, Dreano Y, Mercier A, Thuillez C, et al: Epoxyeicosatrienoic acids contribute with altered nitric oxide and endothelin-1 pathways to conduit artery endothelial dysfunction in essential hypertension. Circulation 125: 1266-1275, 2012.

8. Bellien J, Joannides R, Richard V and Thuillez C: Modulation of cytochrome-derived epoxyeicosatrienoic acids pathway: A promising pharmacological approach to prevent endothelial dysfunction in cardiovascular diseases? Pharmacol Ther 131: $1-17,2011$.

9. Modena MG, Bonetti L, Coppi F, Bursi F and Rossi R: Prognostic role of reversible endothelial dysfunction in hypertensive postmenopausal women. J Am Coll Cardiol 40: 505-510, 2002.

10. Virdis A, Ghiadoni L, Versari D, Giannarelli C, Salvetti A and Taddei S: Endothelial function assessment in complicated hypertension. Curr Pharm Des 14: 1761-1770, 2008.

11. Wallace SM, Yasmin, McEniery CM, Mäki-Petäjä KM, Booth AD, Cockcroft JR and Wilkinson IB: Isolated systolic hypertension is characterized by increased aortic stiffness and endothelial dysfunction. Hypertension 50: 228-233, 2007.

12. Ma Y, Yabluchanskiy A, Lindsey ML and Chilton RJ: Is isolated systolic hypertension worse than combined systolic/diastolic hypertension? J Clin Hypertens (Greenwich) 14: 808-809, 2012.

13. Bellien J, Remy-Jouet I, Iacob M, Blot E, Mercier A, Lucas D, Dreano Y, Gutierrez L, Donnadieu N, Thuillez C, et al: Impaired role of epoxyeicosatrienoic acids in the regulation of basal conduit artery diameter during essential hypertension. Hypertension 60 : 1415-1421, 2012

14. Kim KY, Shin HK, Choi JM and Hong KW: Inhibition of lipopolysaccharide-induced apoptosis by cilostazol in human umbilica vein endothelial cells. J Pharmacol Exp Ther 300: 709-715, 2002.

15. Ge J, Han Y, Jiang H, Sun B, Chen J, Zhang S and Du Z; RACTS (Randomized Prospective Antiplatelet Trial of Cilostazol Versus Ticlopidine in Patients Undergoing Coronary Stenting) Trial Investigators: RACTS: a prospective randomized antiplatelet trial of cilostazol versus ticlopidine in patients undergoing coronary stenting: long-term clinical and angiographic outcome. J Cardiovasc Pharmacol 46: 162-166, 2005.

16. Azuma M, Houchi H, Mizuta M, Kinoshita M, Teraoka K and Minakuchi K: Inhibitory action of cilostazol, a phosphodiesterase III inhibitor, on catecholamine secretion from cultured bovine adrenal chromaffin cells. J Cardiovasc Pharmacol 41 (Suppl 1): S29-S32, 2003.

17. Chao TH, Tseng SY, Li YH, Liu PY, Cho CL, Shi GY, Wu HL and Chen JH: A novel vasculo-angiogenic effect of cilostazol mediated by cross-talk between multiple signalling pathways including the ERK/p38 MAPK signalling transduction cascade. Clin Sci (Lond) 123: 147-159, 2012

18. Park SY, Lee JH, Kim CD, Lee WS, Park WS, Han J, Kwak YG, Kim KY and Hong KW: Cilostazol suppresses superoxide production and expression of adhesion molecules in human endothelial cells via mediation of cAMP-dependent protein kinase-mediated maxi-K channel activation. J Pharmacol Exp Ther 317: 1238-1245, 2006.
19. Shin HK, Kim YK, Kim KY, Lee JH and Hong KW: Remnant lipoprotein particles induce apoptosis in endothelial cells by NAD (P)H oxidase-mediated production of superoxide and cytokines via lectin-like oxidized low-density lipoprotein receptor-1 activation: Prevention by cilostazol. Circulation 109: 1022-1028, 2004

20. Lim JH, Woo JS and Shin YW: Cilostazol protects endothelial cells against lipopolysaccharide-induced apoptosis through ERK1/2- and P38 MAPK-dependent pathways. Korean J Intern Med 24: 113-122, 2009.

21. Franke TF, Kaplan DR and Cantley LC: PI3K: Downstream AKTion blocks apoptosis. Cell 88: 435-437, 1997.

22. McGee MA and Abdel-Rahman AA: Enhanced vascular PI3K/Akt-NOX signaling underlies the peripheral NMDAR-mediated pressor response in conscious rats. J Cardiovasc Pharmacol 63: 395-405, 2014

23. Xu MC, Shi HM, Wang H and Gao XF: Salidroside protects against hydrogen peroxide-induced injury in HUVECs via the regulation of REDD1 and mTOR activation. Mol Med Rep 8: $147-153,2013$.

24. Yang HY, Bian YF, Zhang HP, Gao F, Xiao CS, Liang B, Li J, Zhang NN and Yang ZM: Angiotensin- (1-7) treatment ameliorates angiotensin II-induced apoptosis of human umbilical vein endothelial cells. Clin Exp Pharmacol Physiol 39: 1004-1010, 2012.

25. Zhang XS, Ren JH, Lu JP and Fan Y: Atorvastatin protects against angiotensin II-induced injury and dysfunction in human umbilical vein endothelial cells through bradykinin 2 receptors. J Cardiovasc Pharmacol 56: 171-176, 2010.

26. Wang Q, Zhang M, Ding Y, Wang Q, Zhang W, Song P and Zou MH: Activation of NAD (P)H oxidase by tryptophan-derived 3-hydroxykynurenine accelerates endothelial apoptosis and dysfunction in vivo. Circ Res 114: 480-492, 2014.

27. Urso $C$ and Caimi G: Oxidative stress and endothelial dysfunction. Minerva Med 102: 59-77, 2011 (In Italian).

28. Xu Y, Ruan S, Xie H and Lin J: Role of LOX-1 in Ang II-induced oxidative functional damage in renal tubular epithelial cells. Int J Mol Med 26: 679-690, 2010.

29. Lee YH, Marquez AP, Mungunsukh O and Day RM: Hepatocyte growth factor inhibits apoptosis by the profibrotic factor angiotensin II via extracellular signal-regulated kinase 1/2 in endothelial cells and tissue explants. Mol Biol Cell 21: 4240-4250, 2010

30. Riwanto M, Rohrer L, Roschitzki B, Besler C, Mocharla P, Mueller M, Perisa D, Heinrich K, Altwegg L, von Eckardstein A, et al: Altered activation of endothelial anti- and proapoptotic pathways by high-density lipoprotein from patients with coronary artery disease: Role of high-density lipoprotein-proteome remodeling. Circulation 127: 891-904, 2013.

31. National Research Council: Guide for the Care and Use of Laboratory Animals. 8th edition. National Academy Press, Washington, DC, 1996.

32. Cassis LA, Marshall DE, Fettinger MJ, Rosenbluth B and Lodder RA: Mechanisms contributing to angiotensin II regulation of body weight. Am J Physiol 274: E867-E876, 1998.

33. Su F, Shi M, Yan Z, Ou D, Li J, Lu Z and Zheng Q: Simvastatin modulates remodeling of Kv4.3 expression in rat hypertrophied cardiomyocytes. Int J Biol Sci 8: 236-248, 2012.

34. Wang S, Zhang M, Liang B, Xu J, Xie Z, Liu C, Viollet B, Yan D and Zou MH: AMPKalpha2 deletion causes aberrant expression and activation of NAD (P)H oxidase and consequent endothelial dysfunction in vivo: Role of $26 \mathrm{~S}$ proteasomes. Circ Res 106: 1117-1128, 2010.

35. Giachini FR, Osmond DA, Zhang S, Carneiro FS, Lima VV, Inscho EW, Webb RC, Tostes RC. Clopidogrel, independent of vascular P2Y12 receptor, improves the arterial function in small mesenteric arteries from Ang II-hypertensive rats. Clin Sci (Lond) 118 (7):463-71, 2010.

36. Sanchez M, Lodi F, Vera R, Villar IC, Cogolludo A, Jimenez R, Moreno L, Romero M, Tamargo J, Perez-Vizcaino F, et al: Quercetin and isorhamnetin prevent endothelial dysfunction, superoxide production, and overexpression of p47phox induced by angiotensin II in rat aorta. J Nutr 137: 910-915, 2007.

37. Li R, Wang WQ, Zhang H, Yang X, Fan Q, Christopher TA, Lopez BL, Tao L, Goldstein BJ, Gao F, et al: Adiponectin improves endothelial function in hyperlipidemic rats by reducing oxidative/nitrative stress and differential regulation of eNOS/iNOS activity. Am J Physiol Endocrinol Metab 293: E1703-E1708, 2007. 
38. Gonzalez AA, Green T, Luffman C, Bourgeois CR, Navar LG, Prieto MC. Renal medullary cyclooxygenase-2 and (pro)renin receptor expression during angiotensin II-dependent hypertension. Am J Physiol Renal Physiol. 307 (8): F962-70, 2014.

39. Pezeshki Z, Eshraghi-Jazi F and Nematbakhsh M: Vascular response to graded angiotensin II infusion in offspring subjected to high-salt drinking water during pregnancy: The effect of blood pressure, heart rate, urine output, endothelial permeability, and gender. Int J Vasc Med 2014: 876527, 2014

40. Xu Z, Lu G and Wu F: Simvastatin suppresses homocysteine-induced apoptosis in endothelial cells: Roles of caspase-3, cIAP-1 and cIAP-2. Hypertens Res 32: 375-380, 2009.

41. Dimmeler S and Zeiher AM: Endothelial cell apoptosis in angiogenesis and vessel regression. Circ Res 87: 434-439, 2000.

42. Vergeade A, Mulder P, Vendeville C, Ventura-Clapier R, Thuillez C and Monteil C: Xanthine oxidase contributes to mitochondrial ROS generation in an experimental model of cocaine-induced diastolic dysfunction. J Cardiovasc Pharmacol 60: 538-543, 2012.

43. Dharmashankar K and Widlansky ME: Vascular endothelial function and hypertension: Insights and directions. Curr Hypertens Rep 12: 448-455, 2010.

44. Schäfer SC, Pellegrin M, Wyss C, Aubert JF, Nussberger J, Hayoz D, Lehr HA and Mazzolai L: Intravital microscopy reveals endothelial dysfunction in resistance arterioles in Angiotensin II-induced hypertension. Hypertens Res 35: 855-861, 2012.

45. Daugherty A, Manning MW and Cassis LA: Angiotensin II promotes atherosclerotic lesions and aneurysms in apolipoprotein E-deficient mice. J Clin Invest 105: 1605-1612, 2000.

46. Caplan BA and Schwartz CJ: Increased endothelial cell turnover in areas of in vivo Evans Blue uptake in the pig aorta. Atherosclerosis 17: 401-417, 1973.

47. Liu T, Shen D, Xing S, Chen J, Yu Z, Wang J, Wu B, Chi H, Zhao H Liang Z, et al: Attenuation of exogenous angiotensin II stress-induced damage and apoptosis in human vascular endothelial cells via microRNA-155 expression. Int J Mol Med 31: 188-196, 2013.

48. Marampon F, Gravina GL, Scarsella L, Festuccia C, Lovat F, Ciccarelli C, Zani BM, Polidoro L, Grassi D, Desideri G, et al: Angiotensin-converting-enzyme inhibition counteracts angiotensin II-mediated endothelial cell dysfunction by modulating the p38/SirT1 axis. J Hypertens 31: 1972-1983, 2013.

49. Nako H, Kataoka K, Koibuchi N, Dong YF, Toyama K, Yamamoto E, Yasuda O, Ichijo H, Ogawa $\mathrm{H}$ and Kim-Mitsuyama S: Novel mechanism of angiotensin II-induced cardiac injury in hypertensive rats: The critical role of ASK1 and VEGF. Hypertens Res 35: 194-200, 2012
50. Chen J, Chen W, Zhu M, Zhu Y, Yin H and Tan Z: Propofol attenuates angiotensin II-induced apoptosis in human coronary artery endothelial cells. Br J Anaesth 107: 525-532, 2011.

51. Kröller-Schön S, Jansen T, Schüler A, Oelze M, Wenzel P, Hausding M, Kerahrodi JG, Beisele M, Lackner KJ, Daiber A, et al: Peroxisome proliferator-activated receptor $\gamma$ coactivator $1 \alpha$ deletion induces angiotensin II-associated vascular dysfunction by increasing mitochondrial oxidative stress and vascular inflammation. Arterioscler Thromb Vasc Biol 33: 1928-1935, 2013.

52. Gealekman O, Abassi Z, Rubinstein I, Winaver $\mathrm{J}$ and Binah O: Role of myocardial inducible nitric oxide synthase in contractile dysfunction and beta-adrenergic hyporesponsiveness in rats with experimental volume-overload heart failure. Circulation 105: 236-243, 2002.

53. Chen F, Wu JL, Fu GS, Mou Y and $\mathrm{Hu}$ SJ: Chronic treatment with qiliqiangxin ameliorates aortic endothelial cell dysfunction in diabetic rats. J Cardiovasc Pharmacol Ther 20: 230-40, 2015.

54. Kossmann S, Hu H, Steven S, Schönfelder T, Fraccarollo D, Mikhed Y, Brähler M, Knorr M, Brandt M, Karbach SH, et al: Inflammatory monocytes determine endothelial nitric-oxide synthase uncoupling and nitro-oxidative stress induced by angiotensin II. J Biol Chem 289: 27540-27550, 2014.

55. Napoli C, Paolisso G, Casamassimi A, Al-Omran M, Barbieri M, Sommese L, Infante T and Ignarro LJ: Effects of nitric oxide on cell proliferation: Novel insights. J Am Coll Cardiol 62: 89-95, 2013

56. Laskin DL and Pendino KJ: Macrophages and inflammatory mediators in tissue injury. Annu Rev Pharmacol Toxicol 35: 655-677, 1995

57. Zhang W, Fu F, Tie R, Liang X, Tian F, Xing W, Li J, Ji L, Xing J, Sun X, et al: Alpha-linolenic acid intake prevents endothelial dysfunction in high-fat diet-fed streptozotocin rats and underlying mechanisms. Vasa 42: 421-428, 2013.

58. Yu J, Li M, Qu Z, Yan D, Li D and Ruan Q: SDF-1/CXCR4-mediated migration of transplanted bone marrow stromal cells toward areas of heart myocardial infarction through activation of PI3K/Akt. J Cardiovasc Pharmacol 55: 496-505, 2010.

59. Zheng H, Fu G, Dai T and Huang H: Migration of endothelial progenitor cells mediated by stromal cell-derived factor-1alpha/CXCR4 via PI3K/Akt/eNOS signal transduction pathway. J Cardiovasc Pharmacol 50: 274-280, 2007. 